\title{
A Hybrid Approach for Dehazing Images
}

\author{
Aditi $^{1}$, Shailender Gupta ${ }^{2}$ and Mansi Dua ${ }^{3}$ \\ YMCA University of Science and Technology ${ }^{1,2}$ \\ aditi22391@gmail.com ${ }^{1}$, shailender81@gmail.com ${ }^{2}$, mansidua.15489@gmail.com ${ }^{3}$
}

\begin{abstract}
Bad weather conditions including haze, fog, rain etc. degrade the picture quality which causes poor visualizations. Various methods have been proposed to eradicate the effects of above conditions. Initially, polarization filters were used to restore the foggy image Though this method estimates airlight to quite good an extent but at the same time uses multiple images therefore, cannot be applied on dynamic scenes. In addition, it has very high time complexity. To overcome the use of multiple images Dark chamel prior technique was used that effectively calculates the transmission map to restore the foggy image. For this purpose it requires usage of soft matting technique that causes halo effect and has high time complexity. We are of the opinion that if transmission map is colculated using cark channel prior and airlight is estimated using polarisation filters, the resultant process will not only have lower time complexity but at the same time overçones the drawback of multiple images. The above mentioned technique is implemented in MATLAB-9 andanalysed in this paper. The results shows that usage of this hybrid approach gives quitê better results.
\end{abstract}

Keywords: Single image dehazing, Dark chmnel prior, polarization filters.

\section{Introduction}

Atmospheric visibility can be affected by a number of reasons that may include the presence of bad weather which includes fog, haze, mist, smoke etc. Poor visibility [1-3] not only degrades perceptual image quality but at the same time affects the performance of the computer vis on algorithms uch as surveillance, tracking and navigation. The fog present in images is the conbination of two fundamental phenomena: 'attenuation' and 'airlight' [4]. We firstly explain in brief these two aspects and then describe their combined effect i. e. effect of fog on images.

\subsection{Direct attenuation}

Light beam coming from a scene point, gets attenuated because of scattering by atmospheric particles. This phenomenon is termed as attenuation which reduces contrast in the scene. It is the function of the distance between camera and object. It describes scene radiance and its decay in the medium. It is a multiplicative distortion of the scene radiance. (refer equation 1.1)

Where

$$
\begin{aligned}
& \text { Direct attenuation, } \mathrm{D}(\mathrm{x})=\mathrm{J}(\mathrm{x}) \cdot \mathrm{t}(\mathrm{x}) \\
& \text { Where } \mathrm{t}(\mathrm{x})=\mathrm{e}^{-\beta \mathrm{d}(\mathrm{x})}
\end{aligned}
$$

$J(x)$ is scene radiance

$t(x)$ is transmission map

$\beta$ is atmospheric attenuation coefficient 
$d(x)$ is the distance between an object in the image and observer.

\subsection{Airlight}

Light coming from the source is scattered towards camera and adds whiteness in the scene, this phenomenon is termed as 'airlight'. It is also the function of the distance between camera and object.it results from the previously scattered light and leads to the shift of the scene colors. It is the additive distortion of the scene radiance. Here, the symbols have the usual meaning as described above. ( refer equation 1.2)

\section{Here A is Global atmospheric Light}

$$
\text { Airlight }=A^{*}(1-t(x))
$$

\section{2}

\subsection{Effect Of Fog}

Now, the effect of fog can be defined as the combined effect of both the phenomenon ( refer equation 1.3)

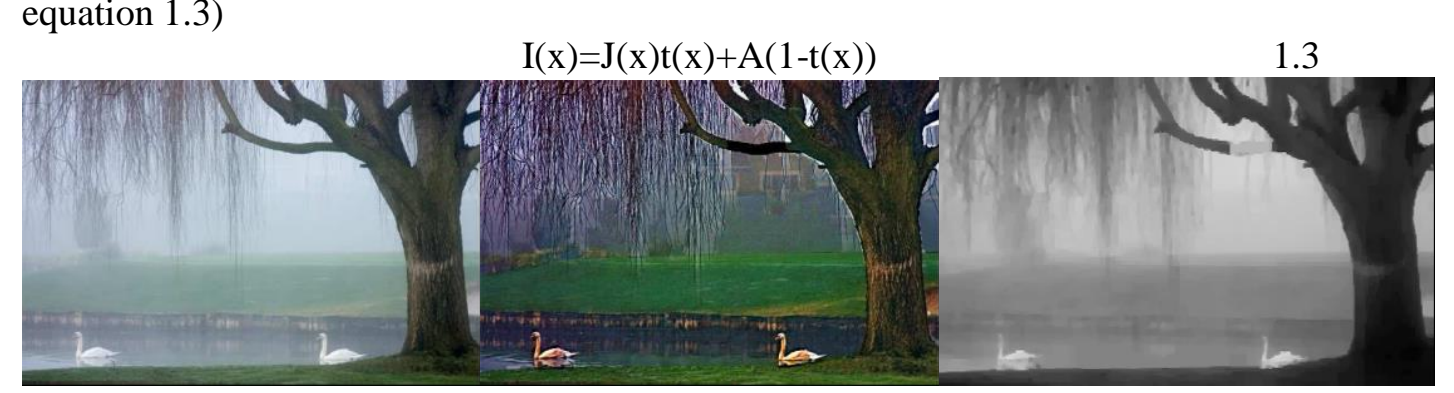

Figure 1 (a) Effect of Fog Figure 1(b) Effect of Aftenuation Figure 1(c) Effect of Airlight

\section{Figure 1. Effect of Air light and Attenuation on Image}

.Here, the Figure 1(a) descr bes the natural image i.e., in presence of fog while the second (see Figure 1(b)) shows the effect of direct attenuation and the third(see Figure 1(c)) describes the effect of airlight.

It is quite clear from the above equations that to remove haze or fog from images requires estimation of transmission map and airlight map. Therefore to minimise the effect of fog various methods have been proposed as explained in the next section.

\subsection{Literature Survey and Problem Definition}

This paper considers two approaches for fog removal as follows:

1.4.1. Polarisation filters: In the initial stages polarisation filters were used to restore the foggy image [5-8]. The polarisation filters involves the calculation of the airlight component using the best and worst state of the polarizer. The best state is defined as the state when the arrigh component contribution is least and vice versa. Using these different degrees of polarisation the intensity of the image can be easily calculated. This method no doubt improves the contrast to a large extent but at the same time has high value of error due to the moving objects. In addition, it is also found that the time complexity of this process is high, mainly due to the use of multiple images for estimation of transmission map. Also, a lot of manual work is required to prepare suitable images under different conditions. Hence, this method cannot be applied to dynamic scenes as such without any modification. 
Later on, new techniques were proposed which instead of working on multiple images, worked on single images such as 3D methods (also known as geometrical methods), Tan et. al. and several other methods [9-16],have been proposed. These methods used single images and demanded approximated 3D geometrical model of the input scene. These resolved the requirement of multiple images but were problematic because the scenes in real world are changing significantly every second [9-10].

1.4.2. Dark Channel Prior (DCP) Technique: In the recent past [15] He et. al. proposed a method that made use of a new concept known as DCP which is applicable on a single image. The paper involves the calculation of two parameters i.e. Airlight and Transmission Map. For the calculation of transmission map DCP technique is used. On the other hand to calculate airlight, the concept described by Tan [12] is used. On analysing it is found that the time complexity of Tan method is very high. In addition, the transmission map requires refinenient using soft matting technique for better results.

We are of the opinion that if the two strategies i.e. DCP and polarisation filters techniques are combined, then we would certainly obtain much better results. Since it is well known that to refine an image we need to estimate transmission map and airlight map. The transmission map is more accurate in case of DCP technique while airlight calculation is better in case of polarization filter. Hence, the hybrid technique to recover a dehazed scene gives far better results.

The rest of the paper is organised as folloys:, section 2 deals with the proposal in mathematical terms, section 3 gives the detalls of the setup parameters used, section 4 provides the results and comparison and section 5 provides us the conclusion of the paper.

\section{The Proposal}

\subsection{Flowchart}

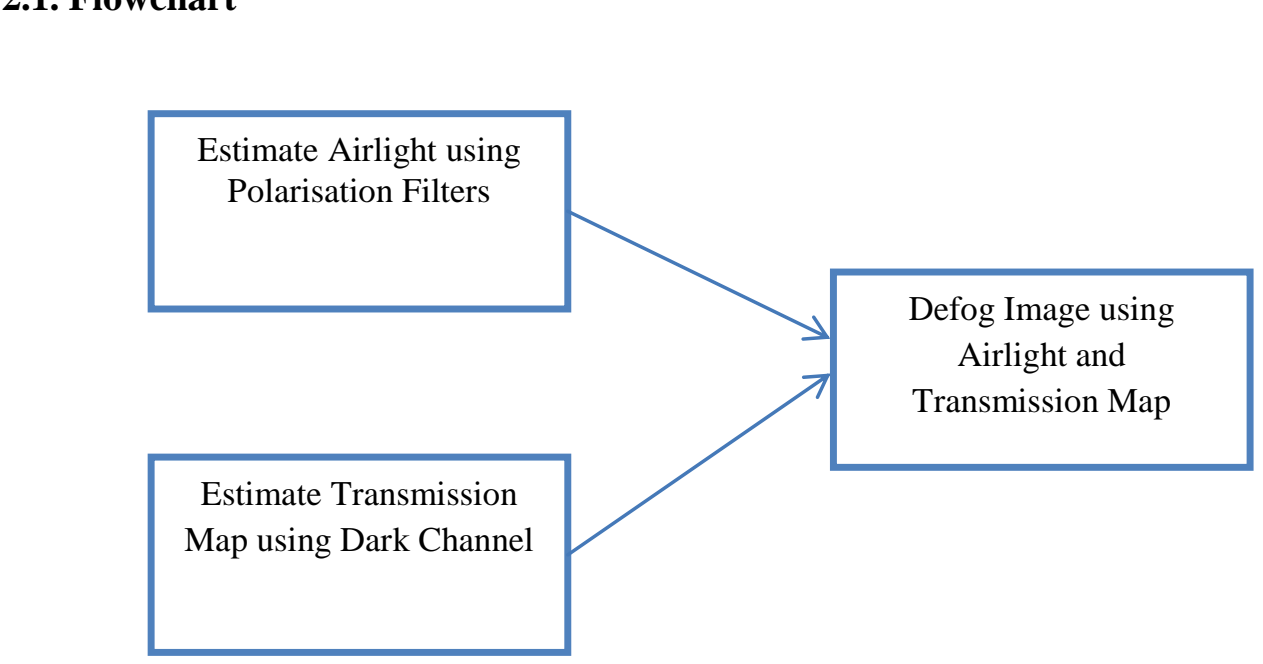

Figure 2. Block Diagram of the Proposal

Figure 2 shows the hybrid technique used to remove fog from images. To remove fog from images we estimate the transmission map using DCP technique and airlight is estimated using 
polarisation filters. The transmission map and airlight map are then used to dehaze the foggy image. These steps are explained in detail in the subsequent subsections.

\subsection{Concept of dark channel}

Initially, the hazy image may be defined as the combination of direct attenuation and airlight components as [15] described by equation 2.1 :

\section{Where}

$$
\mathrm{I}(\mathrm{x})=\mathrm{J}(\mathrm{x}) \mathrm{t}(\mathrm{x})+\mathrm{A}(1-\mathrm{t}(\mathrm{x})) \text {, }
$$

I is the observed intensity

$J$ is the scene radiance

A is the global atmospheric light

$t$ is the medium transmission describing the portion of the light that is not scattered and reaches the camera When the atmosphere is homogenous.

The transmission $\mathrm{t}$ is expressed by equation 2.2 as :

$$
t(x)=e^{-\beta d(x)}
$$

where $\beta$ is the scattering coefficient of the atmosphere and $d$ is the scene depth. Equation (2.2) indicates that the scene radiance is attenuated exponentially with the depth.

For an arbitrary image $\mathbf{J}$, its dark channel $\mathbf{J}^{\text {dark }}$ is defined as (refes equation 2.3 ):

$$
\mathrm{J}^{\mathrm{dark}}(\mathrm{x})=\min _{\mathrm{y} \in \Omega(\mathrm{x})}\left(\min _{\mathrm{i} \in \mathrm{r}, \mathrm{g}, \mathrm{b}\}} \mathrm{J}^{\mathrm{c}}(\mathrm{y})\right)
$$

Except for the sky region, the intensity of $J$ s darkchannel is low and tends to zero known as DCP (see equation 2.4)
2.3 Estimating the transmission map using

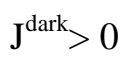
Assumption: Atmospheric light A is def ned as the highest pixel in the image. The first step now is to normalize the haze imaging equation (2.1) by A (refer equation 2.5):

$$
\mathrm{I}^{\mathrm{c}}(\mathrm{x}) / \mathrm{A}^{\mathrm{c}}=\mathrm{t}(\mathrm{x}) \mathrm{J}^{\mathrm{c}}(\mathrm{x}) / \mathrm{A}^{\mathrm{c}}+1-\mathrm{t}(\mathrm{x})
$$
Here, each color channel is normallyzed individually.
Equivalently, by putting the minimum operators on both sides (see equation 2.6):
$\min _{\mathrm{y} \in \Omega(\mathrm{x})}\left(\min _{\mathrm{c}}\left(\mathrm{I}^{\mathrm{c}}(\mathrm{x}) / \mathrm{A}^{\mathrm{c}}\right)\right)=\mathrm{t}^{\prime}(\mathrm{x}) \min _{\mathrm{y} \in \Omega(\mathrm{x})}\left(\min _{\mathrm{c}}\left(\mathrm{J}^{\mathrm{c}}(\mathrm{x}) / \mathrm{A}^{\mathrm{c}}\right)\right)+1-\mathrm{t}^{\prime}(\mathrm{x}) \quad 2.6$

As the scene radiance $\mathrm{J}$ is a haze-free image, the dark channel of $\mathrm{J}$ is close to zero due to the dark channel prior (refer equation 2.7):

As Ac is always positive, this leads to equation 2.8

$$
\mathrm{J}^{\text {dark }}(\mathrm{x})=\min _{\mathrm{y} \in \Omega(\mathrm{x})}\left(\min _{\mathrm{c}} \mathrm{J}^{\mathrm{c}}(\mathrm{y})\right)=0
$$

$$
\min _{\mathrm{y} \in \Omega(\mathrm{x})}\left(\min _{\mathrm{c}} \mathrm{J}^{\mathrm{c}}(\mathrm{y})\right)=0
$$

Patting equation (2.8) into equation (2.6), the multiplicative term can be eliminated and the transmission t' can be estimated as (refer equation 2.9):

$$
\mathrm{t}^{\prime}(\mathrm{x})=1-\min _{\mathrm{y} \in \Omega(\mathrm{x})}\left(\min _{\mathrm{c}}\left(\mathrm{I}^{\mathrm{c}}(\mathrm{x}) / \mathrm{A}^{\mathrm{c}}\right)\right)
$$

The color of the sky in a hazy image I is usually very similar to the atmospheric light A. So in the sky region (refer equation 2.10),

$$
\min _{\mathrm{y} \in \Omega(\mathrm{x})}\left(\min _{\mathrm{c}}\left(\mathrm{I}^{\mathrm{c}}(\mathrm{x}) / \mathrm{A}^{\mathrm{c}}\right)\right)->1
$$

which gives $t^{\prime}(x)->0$. In real world, even on clear days the atmosphere is not absolutely free of any particle, some haze still exists which is a fundamental cue for 
human to perceive depth. This phenomenon is called aerial perspective. So, a very small amount of haze is kept for distant objects by introducing a constant parameter $\mathrm{G}(0<\mathrm{G}$ $\leq 1$ ) into ( equation 2.9) (refer equation 2.11):

$$
\mathrm{t}^{\prime}(\mathrm{x})=1-\boldsymbol{W m i n}_{\mathrm{y} \in \Omega(\mathrm{x})}\left(\min _{\mathrm{c}}\left(\mathrm{I}^{\mathrm{c}}(\mathrm{x}) / \mathrm{A}^{\mathrm{c}}\right)\right)
$$

Here, we fix GO as 0.95 .

\subsection{Estimation of Airlight}

The airlight may be described as the light coming from the source towards the observer. When we use polarization filters, we consider that the airlight is partially polarized. Hence, the polarizer may be modulated to estimate the airlight components. When the polarizer is rotated, we obtain two states where the intensity will be either minimum or maximum. This intensity changes due to the reason that another direction of airlight is filtrated. The orientation where the airlight contribution is most intense, it is denoted by $\mathrm{A}(\mathrm{x})^{\max }$ and where the airlight contribution is least, it is denoted by $\mathrm{A}(\mathrm{x})^{\mathrm{min}}$.

Hence, the airlight $\left(\mathrm{A}_{\text {light }}\right)$ may be expressed by (see equation 2.12):

$$
\mathrm{A}_{\text {light }}=\mathrm{A}(\mathrm{x})^{\max }+\mathrm{A}(\mathrm{x})^{\mathrm{n}}
$$

Here, the assumption is that the direct transmissionis not polanized; its energy is evenly distributed between the polarization components, Hence, the intensity of scene can be expressed as (refer equation 2.13 and equa 1014 ):

$$
\begin{array}{ll}
\mathrm{I}(\mathrm{x})^{\min }=\mathrm{D}(\mathrm{x}) / 2+\mathrm{A}(\mathrm{x})^{\min } & 2.13 \\
\mathrm{I}(\mathrm{x})^{\max }=\mathrm{D}(\mathrm{x}) / 2+\mathrm{A}(\mathrm{x})^{\max } & 2.14
\end{array}
$$

Where $D(x)$ is the direct attenuation

We use the definition of visibility to define the degree of polarization $p$, which defines the airlight degree of polarization as (see equation 2.15):

$$
\mathrm{p}(\mathrm{X}) \mathcal{E}\left(\mathrm{A}(\mathrm{x})^{\max }-\mathrm{A}^{\prime}(\mathrm{x})^{\mathrm{min}}\right) / \mathrm{A}_{\text {light }}
$$

Supposition is that $\mathrm{p}$ is a constant, ranging from 0 to 1 .

So (refer equation 2.16 and equation 2.17),

$$
\begin{aligned}
& \mathrm{A}(\mathrm{x})^{\max }=\mathrm{A}_{\text {light }}(1-\mathrm{p}) / 2 \\
& \mathrm{~A}(\mathrm{x})^{\mathrm{min}}=\mathrm{A}_{\text {light }}(1+\mathrm{p}) / 2
\end{aligned}
$$

Hence, the total intersity can be expressed by equation 2.18 :

\subsection{Image recovery}

$$
\mathrm{I}(\mathrm{x})^{\mathrm{total}}=\mathrm{I}(\mathrm{x})^{\max }+\mathrm{I}(\mathrm{x})^{\min }
$$

For recovering an image $(\mathrm{J}(\mathrm{x}))$, the airlight must be estimated as (see equation 2.19)

$$
\mathrm{A}^{\prime}{ }_{\text {light }}=\left(\mathrm{I}(\mathrm{x})^{\max }-\mathrm{I}(\mathrm{x})^{\mathrm{min}}\right) / \mathrm{p}
$$

Aften this, the transmittance is estimated using dark channel (see equation 2.20)

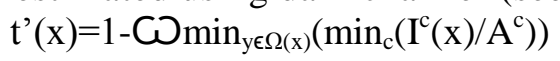

Finally, the image is recovered using the airlight and transmittance (see equation 2.21)

$$
\mathrm{J}^{\prime}(\mathrm{x})=\left(\mathrm{I}(\mathrm{x})^{\text {total }}-\mathrm{A}^{\prime} \text { light }\right) / \mathrm{t}^{\prime}(\mathrm{x})
$$

\section{Simulation setup parameters \\ 3.1 Setup parameters}

The setup parameters are defined as follows in Table 1: 
Table 1. Set Up Parameters

\begin{tabular}{|l|l|}
\hline Size of the image & $204 * 156$ pixels $(8.74 \mathrm{kB}, 9.75 \mathrm{kB}, 22.8 \mathrm{kB}, 32.5 \mathrm{kB})$ \\
\hline $\mathrm{G}$ (Constant Parameter) & 0.95 \\
\hline $\mathrm{D}$ (scene depth) & 1.5 \\
\hline$\beta$ (atmospheric scattering coefficient) & 0.5 \\
\hline $\mathrm{p}$ (degree of polarization) & 0.5 \\
\hline Version of MATLAB & MATLAB 9.0 \\
\hline Size of RAM & 4 GB \\
\hline Configuration of processor used & Intel(R)Core(TM)2 Duo CPU T6500@2.10 GHz \\
\hline
\end{tabular}

\subsection{Performance metrics used}

Various performance metrics used in our experiments are as follows:

1. PSNR (Peak Signal to Noise Ratio) : It is the ratio between the maximum possible power of a signal and the power of corrupting noise that affects the fidelity of its representation. A higher PSNR generally indicates that the reconstruction is of higher quality.

2. MSE( Mean Square Error) : PSNR is most easily defined via the nean squared error (MSE). Given a noise-free $m \times n$ monochrome image I and its noisy approximation K, MSE is defined as:

The PSNR is defined as:

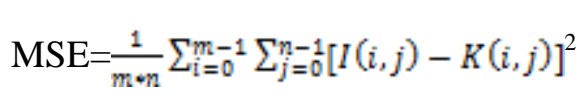

$$
\mathrm{PSNR}=20 \log _{10}(\mathrm{MA} \times \mathrm{X})-\log _{10}(\mathrm{MSE})
$$

3. Time complexity: It is defined as the time jaken by the program to show the output.

4. Qualitative approach; It states the quality to which the extent the picture clarity has been obtained including yarous factors ás contrast.

5. NCD(Normalized Colour Difference) : It is used to measure the degradation in colour quality in colour inages since it approaches the human perception .

\subsection{Snapshots of esults obtained}

Figure 3 to Figure 6 shows the defogging image steps.
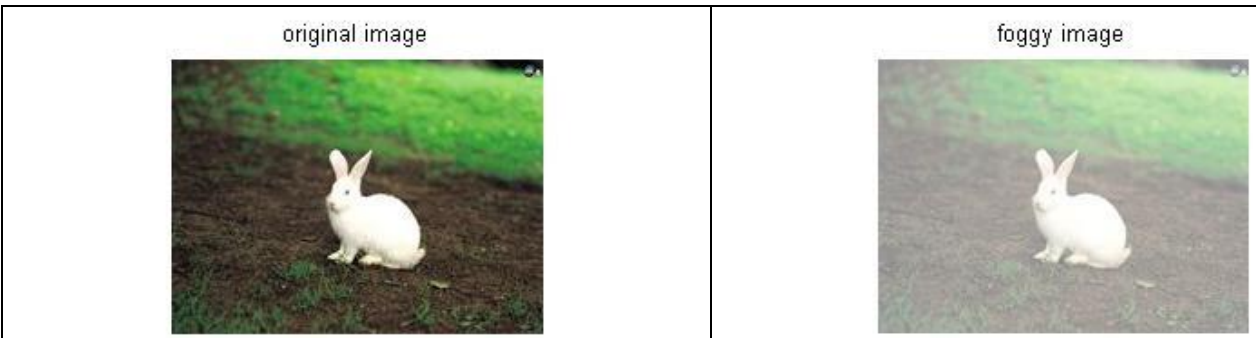

Figure 3 (a) Original Image

Figure 3 (b) Foggy Image 


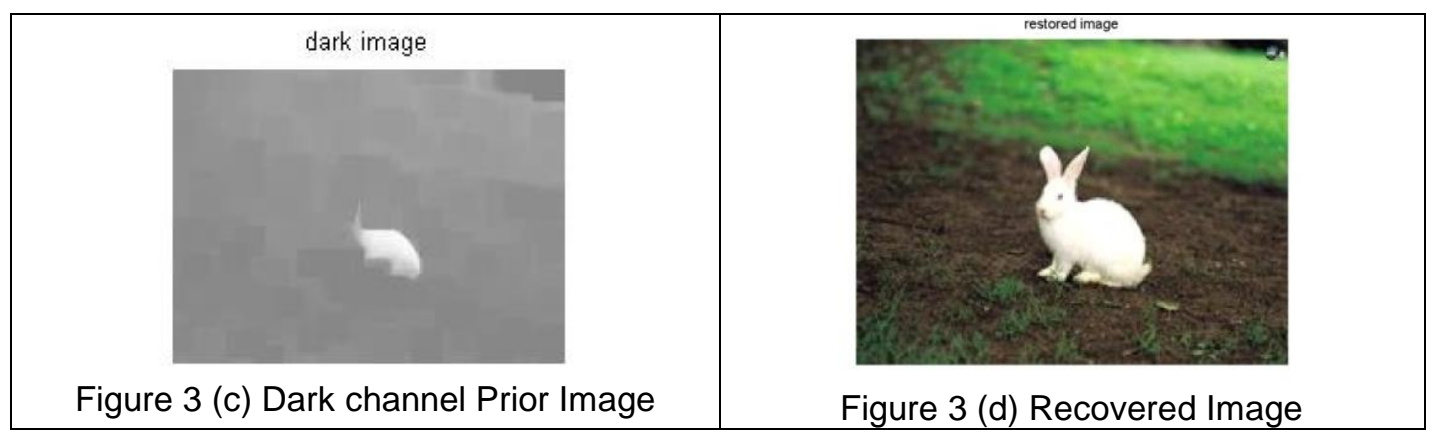

Figure 3. Defogging Steps for Rabbit.jpg

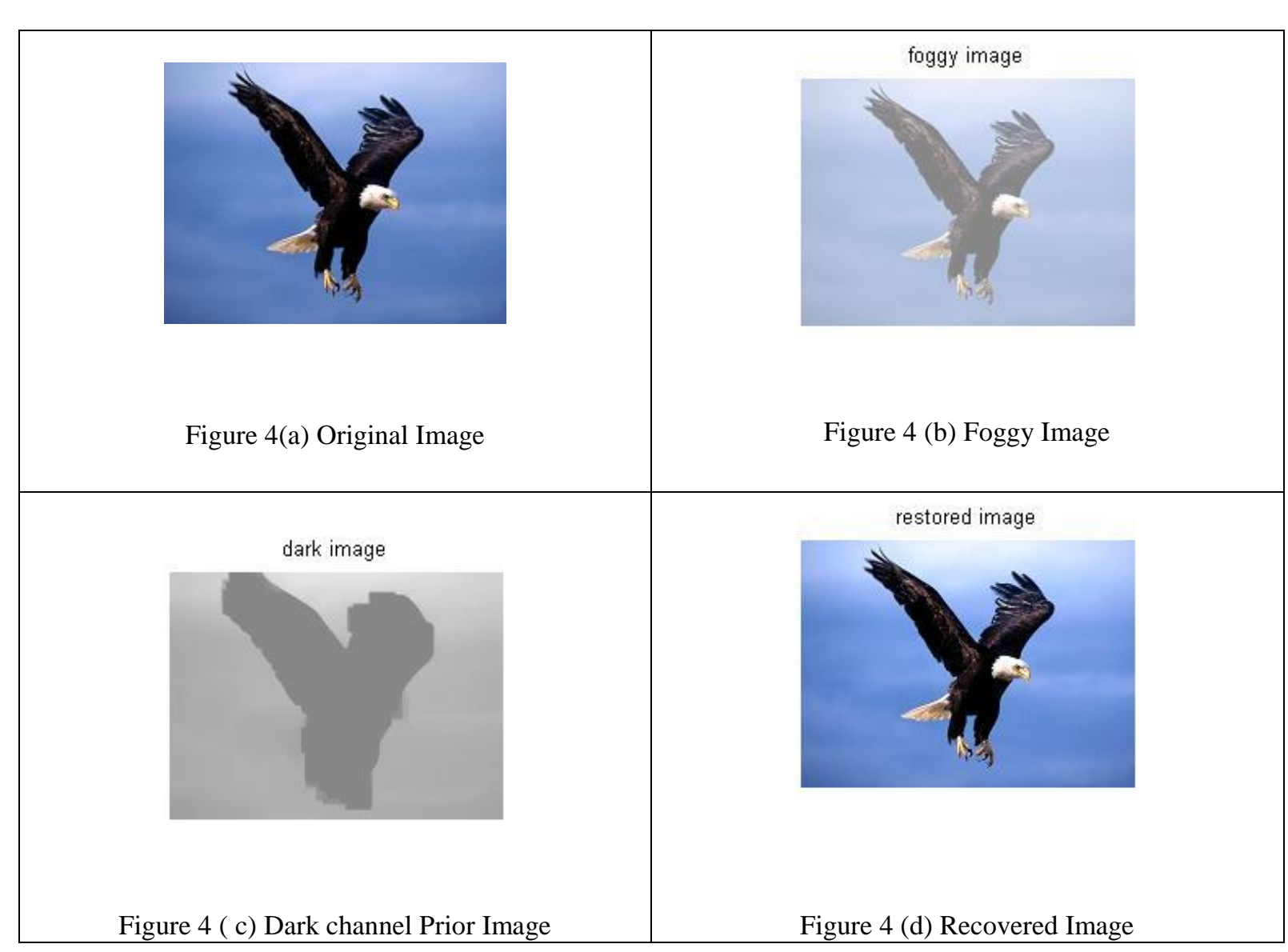

Figure 4. Defogging Steps for eagle.jpg 


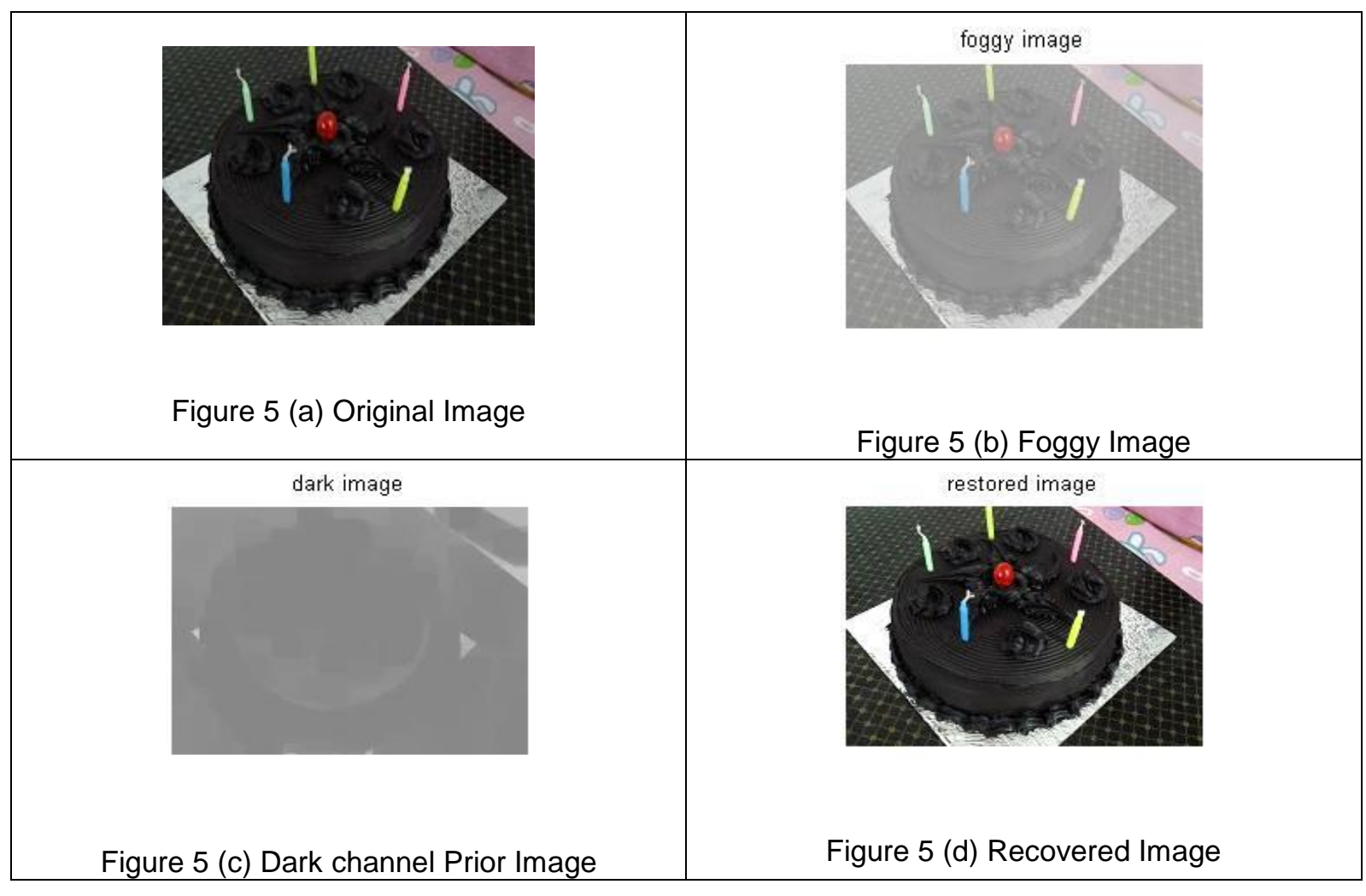

Figure 5. Defogging Steps for birthday_cake.jpg

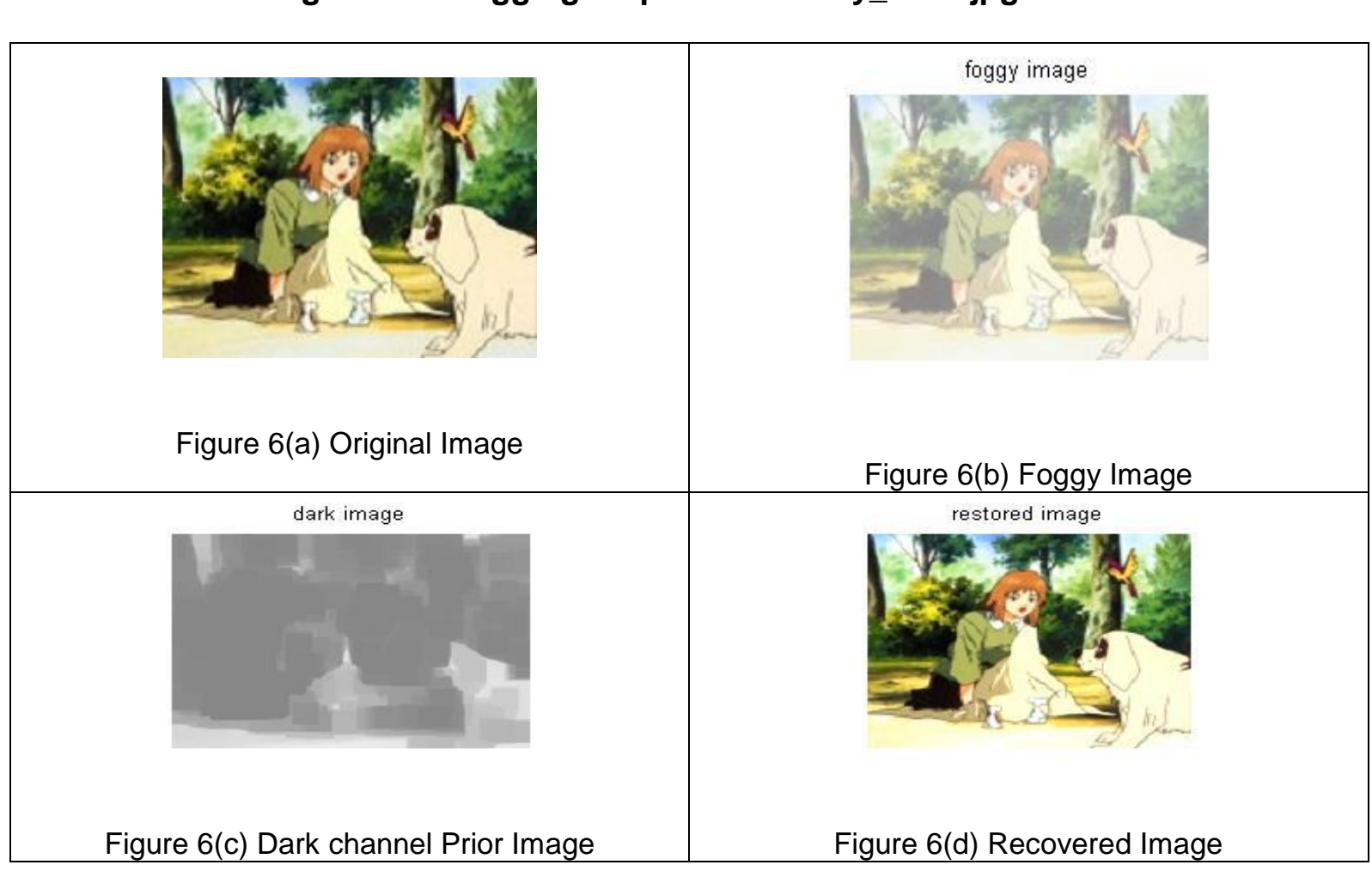

Figure 6. Defogging Steps for snow_white.jpg 


\section{Results}

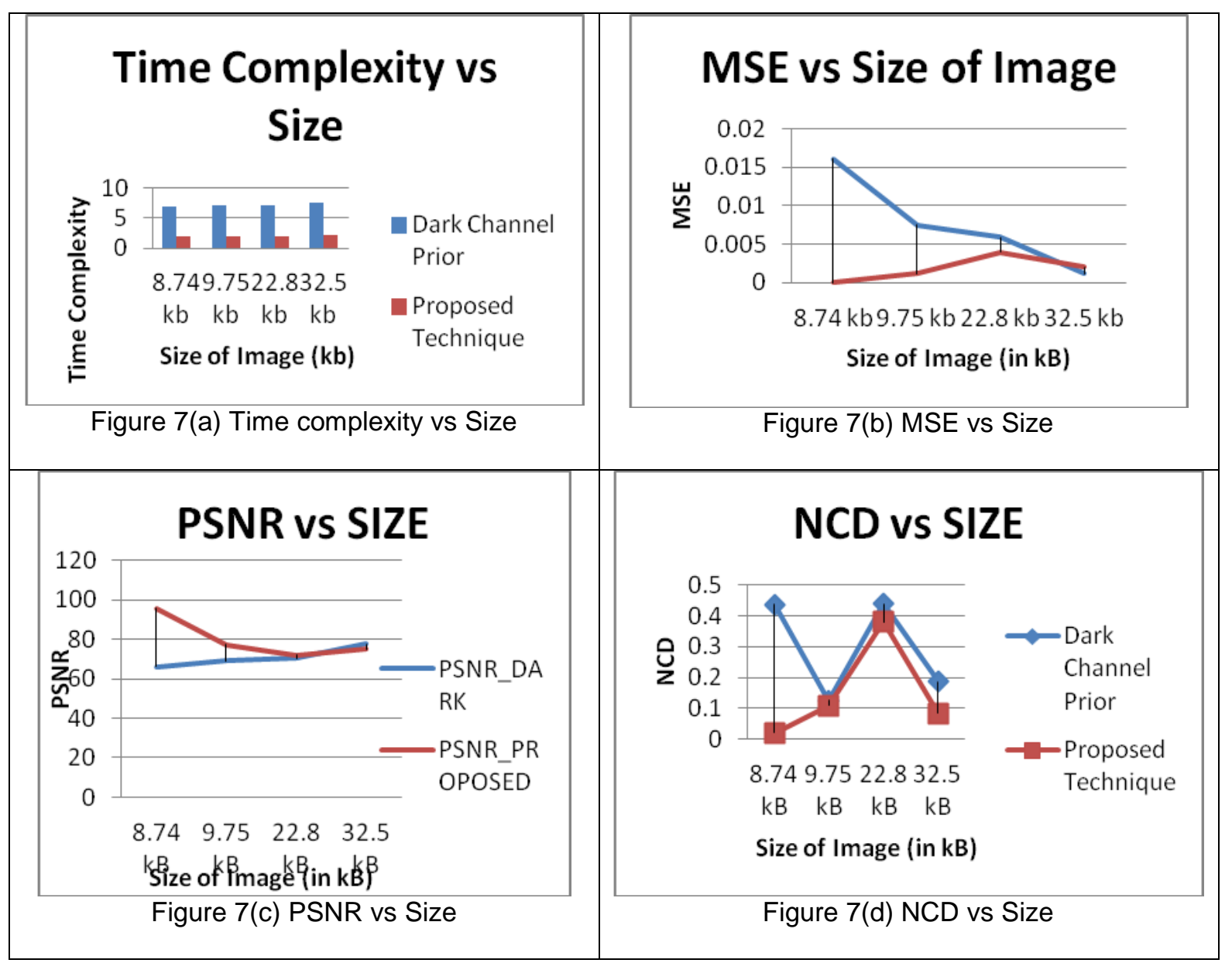

Figure 7. Results of Various Performance Metrics vs Size of Image

The results obtained have been compared with the one obtained through DCP techique[15]. The results are not compred with polarisation filter since it is given in literature that DCP is far better in terms of all performance metrics used in this paper [14-16]

Results of time complexity

Figure 7(a) shows the time complexity results for varying image size. Following inference can be drawn from the results

As the image size increases the time complexity increases for both the techniques.

The proposed technique has very low time complexity in comparison to DCP. The reason for the same is the time consuming step in case of dark channel method and polarisation filters are eliminated from the proposed one.

Results of MSE, PSNR and NCD

Figure 7(b) and 7(c) shows the MSE results for varying image size. Following inference can be drawn from the results 
- $\quad$ As the image size increases the MSE increases, PSNR decreases.

- The proposed technique shows better results of MSE and PSNR for small size images and the results are nearly same for large sized images (see figure 7(b) and 7(c)).

- The value of NCD is always better for the proposed technique (see figure 7(d)).

Table 2. Overall Comparison between the Three Techniques

\begin{tabular}{|l|l|l|l|}
\hline Parameters & Proposed Technique & Dark_Channel & Polarization Filters \\
\hline MSE & Low & High & High \\
\hline PSNR & High & Low & Low \\
\hline NCD & Low & High & High \\
\hline $\begin{array}{l}\text { Multiple Images } \\
\text { Requirement }\end{array}$ & No & No & Yes \\
\hline Time Complexity & Low & Medium & High \\
\hline
\end{tabular}

\section{Analysis of Hybrid Approach}

Table 2 gives the overall comparison of the proposed technique with polarisation filter and DCP technique. The reasons for each parameter are discussed below:

- In case of DCP approach, various assumptions have to be made for estimation of airlight which is not so with polarisation filters. Therefore, this method is better for airlight estimation and also yields better alyes of MSE RidPSNR.

- In case of polarisation filters transmission map is calculated using multiple images which is quite difficult to estimate due to the real life scenario. It can easily be calculated using the DCP technique that provides good results too as given by various researches [1718]. Therefore the results for PSNR and MSE are quite good of our proposed hybrid approach.

- In case of DCP technique, the transmission map estimated is refined using soft matting technique $[15,16]$ as:

$$
\text { ZE(y)= } \mathrm{t}^{\mathrm{T}} \mathrm{Lt}+\lambda\left(\mathrm{t}-\mathrm{t}^{\mathrm{t}}\right)^{\mathrm{T}}\left(\mathrm{t}-\mathrm{t}^{\mathrm{t}}\right)
$$

Where is the vector form of $t, t$ is the vector form of the coarse transmission estimate, and $\lambda$ is a regularization parameter. $L$ is the laplacian matrix and $E$ is the quadratic cost function. The $(i, j)$ element of $L$ is defined as:

$$
\mathcal{L}(i, j)=\sum_{k \mid(i, j) \in w_{k}}\left(\delta_{i j}-\frac{1}{\left|w_{k}\right|}\left(1+\left(\mathbf{I}_{\mathbf{i}}-\mu_{k}\right)^{T}\left(\Sigma_{k}+\frac{\varepsilon}{\left|w_{k}\right|} \mathbf{U}_{3}\right)^{-1}\left(\mathbf{I}_{\mathbf{j}}-\mu_{k}\right)\right)\right)
$$

where $\mathrm{i}, \mathrm{j}$ is the Kronecker delta, $\mu \mathrm{k}$ and $\sum \mathrm{k}$ are the mean and covariance of the colors in window wk centered around $\mathrm{k},|\mathrm{wk}|$ is the number of pixels in each window, and U3 is a $3 \times 3$ identity matrix. $\varepsilon$ is a small regularization parameter.

This is the most time consuming step in case of DCP. While in case of polarisation filters, the use of multiple images in itself is time consuming. These steps were removed from our proposed hybrid approach hence, the results of time complexity are quite good. 


\section{Conclusion}

The proposed technique combines the merits of both the techniques i.e. DCP and Polarisation filters. The following points about our proposed technique can be inferred (see table 4):

1. The time complexity of the method has been reduced to a large extent.

2. The results of MSE and PSNR are better in case of proposed technique as the values obtained are much lower.

3. NCD and contrast of recovered image have been improved in comparison to DCP results.

\section{References}

[1] K. B. Gibson and T. Q. Nguyen, "An analysis of single image defogging methods using a color ellipsoid framework", Gibson and Nguyen EURASIP journal on Image and Video Processing, vøl. 37, (2013), pp.1-14.

[2] P. John, B. Hong, "Correction of simple contrast loss in color images", Pròc. IEE Transactions on Image Processing, vol. 16, no. 2, (2007) February, pp. 511-522.

[3] S. K. Nayar and S. G. Narashiman, "Vision in bad weather", Proc. Seventh IEEE Int'l Conf. Computer Vision, vol. 2, (1999), pp. 820-827.

[4] Y. Jing and Q. Liao, "Fast single image fog removal using edge-preserving smoothing", Proc. IEEE International Conference on Acoustics, Speech and Signal Processing 978-Y-4577-0539-7/11, (2011), pp. 1245-1249.

[5] Y. Y. Schechner, S. G. Narasimahan and S. Nayar, "Instant Dehazing of Images Using Polarization", Proc. IEEE Conf. Computer Vision and Pattern Recognitron, vol. 1, (2001), pp. 325-332.

[6] Y. Y. Schechner, S. G. Narasimahan and S Nayar, "Polarization-based vision through haze", Applied Optics, vol. 42, no. 3, (2003), pp. 511-525.

[7] S. Shwartz, E. Namer and Y. Schechner, "Blind haze separation", Proc. IEEE Int. Conf. Computer Vision, Pattern Recognition, (2006) October, pp.1984-1991

[8] N. Hautiere, J. Tarel and D. Aubert, "Towards fog-free in-vehicle vision systems through contrast restoration", Proc. IEEE Conf Computer Vision and Pattern Recognition, (2007) June, pp. 1-8.

[9] L. B. Wolff and T. E. Boult "Constraining object features using a polarization reflectance model", IEEE Transactions on Pattern Analysis and Machine Intelligence, vol. 13, no. 7, (1991), pp. 635-657.

[10] S. G. Narasimhan and S. K. Nayar, "Mnteractive Deweathering of an image using Physical Models", Proc. IEEE Workshop Color and Photometric Methods on Computer Vision, in Conjunction with IEEE Int'l Conf. Computer vision, (2003) October, pp. 1-8.

[11] J. Oakley and B. Satherley "Improving image quality in poor visibility conditions using a physical model for constant degradation", IEFE Transactions on Image Processing, vol. 7, no. 2, (1998), pp. 167-169.

[12] R. Tan, "Visibility in bad weather from a single image", Proc. IEEE Conf. Computer Vision, Pattern Recognition, (2008) June, pp.1-8.

[13] R. Fattal, "Single imaze dehazing", ACM Transactions on Graphics, vol. 27, (2008) August, pp.1-9.

[14] L. Kratz and K. Nishino, "Factorizing scene albedo and depth from a single foggy image", Proc IEEE Int. Conf. Computer Vision, (2009) September, pp. 1701-1708.

[15] K. He, J. Sum and X. Tang, "Single image haze removal using dark channel prior", IEEE Int. Conf. On Computer Vision and Pattern Recognition, (2009), pp. 1956-1963.

[16] A. Levin, D. Lischinski and Y. Weiss, "A closed form solution to Natural Image Matting", Proc. IEEE Conf. Computer Vision and Pattern Recognition, vol. 1, (2006), pp. 61-68.

[17] A. K. Tripathi and S. Mukhopadhyay, "Single Image Fog Removal using Anisotropic Diffusion", published In IET Image Processing. vol. 6, (2012), pp. 966-975.

[18] J. S.-C. Yuk and K.-Y. K. Wong, "Adaptive Background Defogging with Foreground Decremental Preconditioned Conjugate Gradient”, Proc. Asian Conference on Computer Vision, vol. 4, (2013), pp. 602614. 
International Journal of Hybrid Information Technology

Vol.7, No.3 (2014)

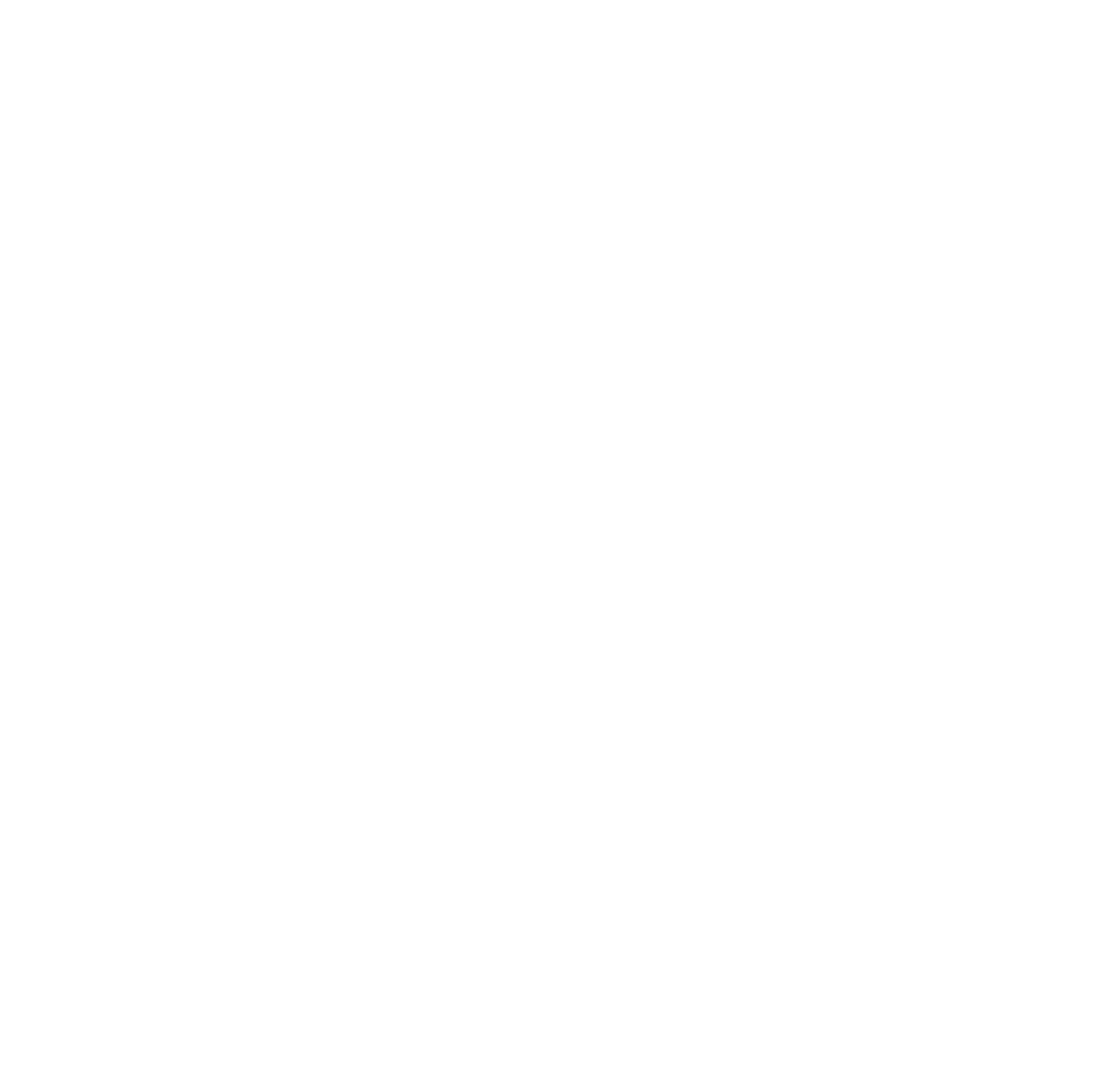

